\title{
Quality of life and mortality among survivors of acute respiratory distress syndrome in South Korea: a nationwide cohort study
}

\author{
Hey-ran $\mathrm{Choi}^{1} \cdot$ In-Ae Song ${ }^{2} \cdot$ Tak Kyu Oh $^{2,3}$ (])
}

Received: 18 October 2021 / Accepted: 1 January 2022 / Published online: 21 January 2022

(c) The Author(s) under exclusive licence to Japanese Society of Anesthesiologists 2022

\begin{abstract}
Purpose Worsening quality of life (QOL) is an important health issue in acute respiratory distress syndrome (ARDS) survivors. We aimed to investigate the prevalence of worsening QOL among ARDS survivors and their association with mortality. Methods South Korean National Health Insurance database information for all adults admitted to intensive care units for ARDS from January 1, 2010 to December 31, 2018 who survived $\geq 365$ days were included in this study.

Results A total of 4452 ARDS survivors were included in the final analysis. Total QOL had worsened in 1667 (37.4\%) of the survivors at the follow-up 1 year after being diagnosed with the syndrome. Specifically, 1298 patients (29.2\%) experienced decreased income, $334(7.5 \%)$ lost their jobs, and $327(7.3 \%)$ had newly acquired disabilities. In the multivariable Cox regression analysis, worsening QOL was not associated with 2-year all-cause mortality among survivors $(P=0.140)$. However, newly acquired disability was associated with 1.74-fold (hazard ratio [HR]: 1.74, 95\% confidence interval [CI] $1.31-2.33 ; P<0.001)$ higher 2-year all-cause mortality, while decreased income $(P=0.571)$ and unemployment $(P=0.952)$ were not associated with it. In addition, newly acquired respiratory disability was associated with a 6.61-fold higher risk of 2-year respiratory mortality (HR: 6.61, 95\% CI 3.14-13.90; $P<0.001$ ).

Conclusions At the 1-year follow-up period, one-third of ARDS survivors experienced worsening QOL in South Korea. Specifically, newly acquired disability was associated with a higher risk of 2-year all-cause and respiratory mortality among patients who survived ARDS.
\end{abstract}

Keywords Critical care $\cdot$ Intensive care units · Quality of life $\cdot$ Mortality $\cdot$ Respiratory distress syndrome

\section{Introduction}

Acute respiratory distress syndrome (ARDS) is a clinical syndrome characterized by acute hypoxemia with bilateral pulmonary infiltration and decreased lung compliance [1]. The prevalence of ARDS in the intensive care unit (ICU)

Hey-ran Choi and In-Ae Song have equal contribution as the cofirst authors.

\section{Tak Kyu Oh}

airohtak@hotmail.com; 66034@snubh.org

1 Department of Anesthesiology and Pain Medicine, Inje University Seoul Paik Hospital, Seoul, South Korea

2 Department of Anesthesiology and Pain Medicine, Seoul National University Bundang Hospital, Gumi-ro, 173, Beon-gil, Bundang-gu, Seongnam 13620, South Korea

3 Department of Anesthesiology and Pain Medicine, College of Medicine, Seoul National University, 103 Daehak-ro, Jongno-gu, Seoul, South Korea was reported as $10.4 \%$ among ICUs in 50 countries [2]. As the mortality rate among ARDS patients varies from 11 to $87 \%$ globally [3], there might be many survivors of ARDS worldwide. Therefore, quality of life (QOL) among survivors of ARDS is a significant public health issue [4].

Previous studies reported that ARDS survivors experienced reduced QOL [5-8], and most studies evaluated the QOL of ARDS survivors using surveys [4]. Recent studies have focused on the QOL of ARDS survivors from various perspectives [9-13], and some researchers have reported that ARDS survivors experienced unemployment, lost earnings, an increased burden of healthcare utilization, and functional disabilities in daily life [10-14]. Although there have been advances in understanding worsening QOL among ARDS survivors, its impact on further prognosis has not yet been identified.

Therefore, we aimed to examine the prevalence of worsening QOL among ARDS survivors as of the 1-year followup period after diagnosis of ARDS. In addition, we also 
examined whether worsening QOL was associated with mortality among the survivors. We hypothesized that ARDS survivors might experience worsening QOL and that it might be associated with poorer long-term survival.

\section{Methods}

\section{Study design, setting, and ethical statement}

The Reporting of Observational Studies in Epidemiology guidelines was followed in this study as a nationwide population-based cohort study in South Korea [15]. The Institutional Review Board (IRB) of Seoul National University Bundang Hospital approved the protocol of this study (Approval Number: X-2008-630-903), and national health insurance service (NHIS) approved data sharing after approval of the study protocol (Approval Number: NHIS2021-1-424). The IRB waived the need for informed consent because we used data that were anonymized and had been extracted retrospectively for analysis in this study.

\section{Data source}

The data were extracted from the NHIS database and Statistics Korea for this study. First, information regarding disease diagnosis and prescription of drugs and/or procedures was extracted from the NHIS database. As a public health insurance system in South Korea, the NHIS contains all disease diagnoses using the International Statistical Classification of Diseases and Related Health Problems-tenth revision (ICD-10) codes and prescription information for patients to receive financial coverage of treatment expenses by the government. We extracted data regarding death from the database of Statistics Korea. As a central government organization, Statistics Korea records national statistics and data regarding accurate dates and causes of death of all individuals in South Korea. Physicians register the principal cause of death for all individuals in the database. All dates and causes of death were collected by December 31, 2020, for this study.

\section{ARDS survivor}

Adult ( $\geq 18$ years old) critically ill patients who were admitted to the ICU between January 1, 2010, and December 31, 2018, and diagnosed with ARDS using ICD-10 codes of J80 were initially screened in this study. As ARDS is a syndrome that can occur with other pathologic conditions [16], we included both the main diagnosis cases and secondary diagnosis cases of ARDS. Therefore, patients with a primary diagnosis of pneumonia and a secondary diagnosis of ARDS were included in this study. The main diagnosis was defined by the NHIS after the end of hospitalization as a certain disease in which the patient's demand for treatment or examination was the greatest during hospitalization. If a patient was admitted to the ICU with a diagnosis of ARDS two times or more during the study period, only the first episode of admission was considered in this study. Those who were alive $\geq 365$ days after their ARDS diagnosis were considered ARDS "survivors".

\section{Quality of life among ARDS survivors}

The QOL of ARDS survivors was evaluated using three methods. First, the household income level at ICU admission for ARDS was compared to the level 1 year later. The NHIS database contains the household income levels of subscribers to determine their insurance premiums. The household income level of all subscribers was determined according to income, living standards, property, and rate of participation in economic activities, and divided into four groups using the quartile ratio. Second, we evaluated unemployment after ARDS treatment. We examined the employment status before and after admission for ARDS treatment among survivors (employment status is registered in the NHIS database; self-employment was not considered). Third, new disabilities acquired within a year of ARDS diagnosis were evaluated. All individuals with any disability must be registered in the NHIS database to receive various benefits from the social welfare system in South Korea. There are 15 types of disabilities, including physical disability, brain lesion disability, visual disturbance, hearing disability, speech disability, intellectual disorder, autism, mental disorders, renal disorders, heart disorders, respiratory disabilities, hepatopathy, facial disfigurement, intestinal and urinary fistulae, and epilepsy. Each disability is determined strictly depending on the law by the specialist physician in each field. Regarding patients' disabilities, each is assigned one of six grades according to severity $(1 \mathrm{st}=$ most severe; 6 th $=$ most mild $)$. In this study, the 1st-3rd grades of disability were considered "severe"; 4th-6th grades were considered "mild to moderate." The criteria for the respiratory disability grading in South Korea are based on the symptoms of dyspnea, chest $\mathrm{X}$-ray imaging, pulmonary function testing, and arterial blood gas analysis [17]; grading criteria are presented in Table S1.

\section{Study endpoints}

The primary endpoint in this study was worsening QOL (decreased household income, unemployment, and newly acquired disability) within 1 year after diagnosis of ARDS among survivors. The secondary endpoint was 2-year allcause mortality among survivors of ARDS. If the main cause of death was respiratory disease (J00-J99), it was classified 
as "respiratory mortality"; all mortality not due to respiratory disease was classified as "other mortality."

\section{Covariates}

Physical variables considered included age and sex; ARDS treatment results variables for survivors were divided into three types: (1) discharge and follow-up at the same hospital, (2) transfer to other long-term facility centers, and (3) discharge and outpatient clinic follow-up. Admitting departments were divided into internal medicine (IM) and non-IM. Length of hospitalization (days) and total cost of hospitalization (USD) were used as covariates. The type of hospital admission was also considered a covariate, and patients were divided into (1) transfer from another hospital, (2) admission through emergency room, or (3) admission through outpatient clinic. As high case volume centers were associated with improved hospital survival outcomes in patients with ARDS [18], we calculated the annual case volume of ARDS treatment in each hospital as a covariate.

The patients were assigned into four groups using quartile ratio, based on the hospital to which patients with ARDS were admitted, such as Q1 $\leq 4$, Q2: 5-14, Q3: 15-28, and $\mathrm{Q} 4 \geq 29$. The main diagnosis of ARDS, diagnoses of shock (R57), and sepsis (A40, A41, and R65.2) were considered covariates. If a patient with ARDS was diagnosed with sepsis as the primary diagnosis, it was considered "sepsisassociated ARDS." Extracorporeal membrane oxygenation (ECMO), neuromuscular blockade (NMB) use, continuous renal replacement therapy (CRRT) use, and experience of cardiopulmonary resuscitation (CPR) were also considered covariates. The Charlson Comorbidity Index (CCI) was also calculated using registered ICD-10 codes of individual underlying diseases within 1 year before admission for ARDS, as shown in Table S2. The CCI was divided into four groups as categorical variables $(0-1,2-3,4-5$, and $\geq 6)$.

\section{Statistical analysis}

The clinicopathological characteristics of total ARDS survivors are presented as numbers with percentages for categorical variables and mean values with standard deviation (SD) for continuous variables. First, QOL before and after ARDS treatment among survivors were examined. Second, QOL worsening according to age, the CCI, the number of days of total mechanical ventilatory support, duration of hospitalization, use of ECMO support, sepsis-associated ARDS, and the experience of CPR among ARDS survivors were examined. For continuous variables (age, CCI, day of total mechanical ventilatory support, and duration of hospitalization), median values were used as cutoffs to categorize the subgroups. Third, we examined worsening QOL among hospital survivors who were discharged after hospitalization for ARDS treatment, because the survivors who were discharged but died within a year after being diagnosed with ARDS, might affect the results. Following this, we performed a multivariable logistic regression analysis for QOL worsening among ARDS survivors. All covariates were included in the multivariate adjustment model. The Hosmer-Lemeshow test was used to confirm the model's goodness of fit, and the results were presented as odds ratios (ORs) with 95\% confidence intervals (CIs).

Next, we constructed multivariable Cox regression models for 2-year all-cause mortality after diagnosis of ARDS among survivors. Three separate models were constructed to avoid multicollinearity. Model 1 examined the association between worsening QOL and the risk of 2-year all-cause mortality. Model 2 examined the association between worsening QOL in detail (decreased income, unemployment, and newly acquired disability) and the risk of 2-year all-cause mortality. Model 3 examined the effect of newly acquired respiratory disability among total disability on the risk of 2-year all-cause mortality.

We performed multivariable Cox regression modeling for 2-year respiratory and other mortality after ARDS diagnosis among survivors. The results of Cox regression analyses were presented as hazard ratios (HRs) with $95 \%$ CIs, and $\log -\log$ plots were used to confirm satisfaction of the central assumption of Cox proportional hazard models. Variance inflation factors $(<2.0)$ confirmed there was no issue of multicollinearity between variables in the model. All statistical analyses were performed using $\mathrm{R}$ software (version 4.0.3, $\mathrm{R}$ packages, R Project for Statistical Computing, Vienna, Austria), and statistical significance was set at $P<0.05$.

\section{Results}

From January 1, 2010, to December 31, 2018, there were 23,280 cases in ICUs for ARDS treatment. After excluding 7407 patients, who were admitted to the ICU with a diagnosis of ARDS from 2010 to 2018 twice or more, 365 pediatric cases ( $<18$ years old), 15,508 adult patients with ARDS were initially screened. Among them, 11,056 patients died within a year of ARDS diagnosis, so 4452 ARDS survivors were included in the final analysis. The 2-year all-cause mortality occurred in $549(12.3 \%)$ patients, and $138(3.1 \%)$ died within 2 years due to respiratory disease, while 411 (9.2\%) patients died due to other causes (Fig. 1). Clinicopathological characteristics are shown in Table 1. The mean age of survivors was 62.9 years (SD: 17.4 years old); 2657 (59.7\%) were male. Among survivors, the mean length of hospitalization was 18.6 days (SD: 15.3 days) and the mean value of total cost for hospitalization was 9714.1 USD (SD: 12,266.7 USD). 


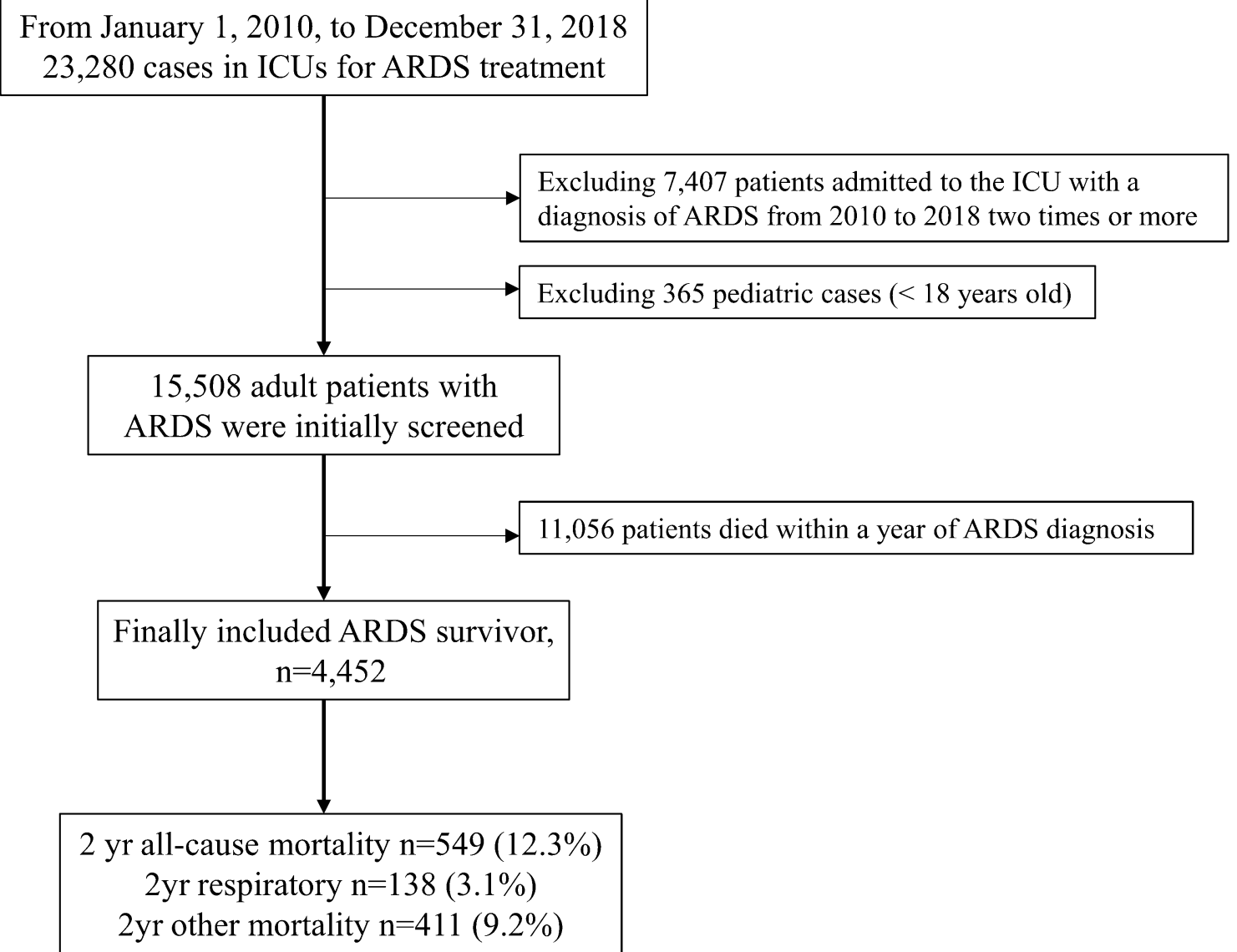

Fig. 1 Flow chart depicting ARDS survivors. ARDS, acute respiratory distress syndrome

\section{QOL among ARDS survivors}

Table 2 shows the QOL before and after ARDS treatment among survivors. Among survivors, 1298 (29.2\%) patients experienced decreased income, 334 patients (7.5\%) lost their jobs, and 327 (7.3\%) had newly acquired disabilities. Among the newly acquired disabilities, $66(1.5 \%)$ had newly acquired respiratory disabilities. In addition, the mild to moderate and severe disability groups increased to $86(1.9 \%)$ and 221 (4.9\%), respectively, after ARDS treatment. Eventually, total QOL worsened in 1667 (37.4\%) ARDS survivors 1 year after their ARDS diagnosis. Table 3 shows the results of the multivariable logistic regression model for QOL worsening among ARDS survivors. Younger age (OR: 0.99, 95\% CI 0.99-0.99; $P<0.001$ ), male sex (vs. female; OR: 1.17 , 95\% CI 1.03-1.32; $P=0.018$ ), and total cost of hospitalization (1000 USD increase; OR, 1.01, 95\% CI 1.01-1.02; $P=0.022)$ were associated with worsening QOL. In addition, survivors who were transferred to other long-term facility centers (OR: $0.74,95 \%$ CI $0.70-0.91 ; P=0.017$ ) or discharged and with follow-ups in outpatient clinics (OR: $0.80,95 \%$ CI $0.70-0.91 ; P=0.001$ ) were associated with a lower incidence of worsening QOL. Table 4 shows the QOL worsening according to age, the CCI, the number of days of total mechanical ventilatory support, duration of hospitalization, use of ECMO support, sepsis-associated ARDS, and experience of CPR among ARDS survivors. Of the ARDS survivors, $43 \%(945 / 2,200)$ of age $<66$-yearold group experienced QOL worsening, while $32.1 \%$ of age $\geq 66$-year-old experienced QOL worsening among ARDS survivors. Table S3 also shows the QOL before and after ARDS treatment among hospital survivors who were discharged alive after hospitalization for ARDS treatment. Of the survivors, $1310(14.5 \%)$ experienced decreased income, 287 (3.2\%) lost their job, and 178 (2.0\%) newly acquired a disability. Thus, the QOL worsened in 1608 (17.8\%) ARDS survivors. However, QOL of 3621 survivors $(40.2 \%$ ) had not been evaluated after discharge because data could not be obtained regarding their income level, job, or disability due to death within a year after being diagnosed with ARDS. 
Table 1 Clinicopathological characteristics

\begin{tabular}{|c|c|}
\hline Variable & Mean $(\mathrm{SD})$ or $N(\%)$ \\
\hline Age, year & $62.9(17.4)$ \\
\hline Sex, male & $2657(59.7)$ \\
\hline \multicolumn{2}{|l|}{ Treatment result } \\
\hline Discharge, and follow-up in same hospital & $1691(38.0)$ \\
\hline Transfer to other long-term facility center & $366(8.2)$ \\
\hline Discharge, and outpatient clinic follow-up & $2395(53.8)$ \\
\hline \multicolumn{2}{|l|}{ Admitting department } \\
\hline IM & $3602(80.9)$ \\
\hline Non-IM & $850(19.1)$ \\
\hline Length of hospitalization, day & $18.6(15.3)$ \\
\hline Total cost for hospitalization, USD & $9714.1(12,266.7)$ \\
\hline Insurance coverage for hospitalization, USD & $8161.4(10,585.2)$ \\
\hline \multicolumn{2}{|l|}{ Hospital admission } \\
\hline Transfer from another hospital & $264(5.9)$ \\
\hline Admission through Emergency Room & $2714(61.0)$ \\
\hline Admission through outpatient clinic & $1474(33.1)$ \\
\hline \multicolumn{2}{|l|}{ Annual case volume of ARDS admission } \\
\hline $\mathrm{Q} 1 \leq 4$ & $1070(24.0)$ \\
\hline Q2: 5-14 & $907(20.4)$ \\
\hline Q3: 15-28 & $1289(29.0)$ \\
\hline Q4 $\geq 28$ & $1186(26.6)$ \\
\hline Main diagnosis of ARDS & $2246(50.4)$ \\
\hline Sepsis-associated ARDS & $526(11.8)$ \\
\hline Diagnosis of shock during hospitalization & $236(5.3)$ \\
\hline CCI at hospital admission for ARDS & $3.3(2.5)$ \\
\hline $0-1$ & $1180(26.5)$ \\
\hline $2-3$ & $1536(34.5)$ \\
\hline $4-5$ & $943(21.2)$ \\
\hline$\geq 6$ & $793(17.8)$ \\
\hline ECMO support & $215(4.8)$ \\
\hline NMB use & $1520(34.1)$ \\
\hline CRRT use & $184(4.1)$ \\
\hline Duration of mechanical ventilator use, day & $5.3(8.1)$ \\
\hline Experience of CPR during hospitalization & $116(2.6)$ \\
\hline \multicolumn{2}{|l|}{ Year of admission for ARDS } \\
\hline 2010 & $602(13.5)$ \\
\hline 2011 & $470(10.6)$ \\
\hline 2012 & $430(9.7)$ \\
\hline 2013 & $361(8.1)$ \\
\hline 2014 & $465(10.4)$ \\
\hline 2015 & $443(10.0)$ \\
\hline 2016 & $613(13.8)$ \\
\hline 2017 & $523(11.7)$ \\
\hline 2018 & $545(12.2)$ \\
\hline
\end{tabular}

SD, standard deviation; IM, internal medicine; ARDS, acute respiratory distress syndrome; CCI, Charlson comorbidity index; ECMO, extracorporeal membrane oxygenation; NMB, neuromuscular blockade; CPR, cardiopulmonary resuscitation
Table 2 QOL before and after ARDS treatment

\begin{tabular}{|c|c|c|}
\hline Variable & Before ARDS & After ARDS \\
\hline Presence of job ${ }^{\mathrm{a}}$ & $2239(49.7)$ & $2294(51.5)$ \\
\hline \multicolumn{3}{|l|}{ Annual income level $^{\mathrm{b}}$} \\
\hline Q1 (lowest) & $1321(29.7)$ & $1380(31.0)$ \\
\hline Q2 & $755(17.0)$ & $682(15.3)$ \\
\hline Q3 & $903(20.3)$ & $882(19.8)$ \\
\hline Q4 (highest) & $1380(31.0)$ & $1354(30.4)$ \\
\hline Unknown & $93(2.1)$ & $154(3.5)$ \\
\hline \multicolumn{3}{|l|}{ Disability $^{c}$} \\
\hline Mild to moderate & $454(10.2)$ & $540(12.1)$ \\
\hline Severe & $568(12.8)$ & 789 (17.7) \\
\hline \multicolumn{3}{|l|}{ Disability type } \\
\hline Physical disability & $402(9.0)$ & $435(9.8)$ \\
\hline Brain lesion disability & $210(4.7)$ & $346(7.8)$ \\
\hline Visual disturbance & $82(1.8)$ & $92(2.1)$ \\
\hline Hearing disability & $98(2.2)$ & $114(2.6)$ \\
\hline Speech disability & $3(0.1)$ & $5(0.1)$ \\
\hline Intellectual disorder & $55(1.2)$ & $60(1.3)$ \\
\hline Autism & $1(0.0)$ & $1(0.0)$ \\
\hline Mental disorder & $38(0.9)$ & $37(0.8)$ \\
\hline Renal disorder & $59(1.3)$ & $99(2.2)$ \\
\hline Heart disorder & $5(0.1)$ & $9(0.2)$ \\
\hline Respiratory disability & $51(1.1)$ & $117(2.6)$ \\
\hline Hepatopathy & $3(0.1)$ & $4(0.1)$ \\
\hline Facial disfigurement & $0(0.0)$ & $0(0.0)$ \\
\hline Intestinal and urinary fistulae & $9(0.2)$ & $12(0.3)$ \\
\hline Epilepsy & $6(0.1)$ & $6(0.1)$ \\
\hline
\end{tabular}

$1298(29.2 \%)$ experienced decreased income, 334 (7.5\%) lost job, and $327(7.3 \%)$ had newly acquired disability. $66(1.5 \%)$ have newly acquired respiratory disability

Total QOL worsening occurred in 1667 (37.4\%) among ARDS survivors

QOL, quality of life; ARDS, acute respiratory distress syndrome

\section{Survival analysis}

Table 5 shows the results of the multivariable Cox regression model for 2-year all-cause mortality after the diagnosis of ARDS. In model 1, worsening of QOL was not associated with 2-year all-cause mortality among survivors (HR: 1.10, 95\% CI 0.92-1.78; $P=0.140)$. All HRs with $95 \%$ CIs of the covariates in Model 1 are presented in Table S4. In model 2, newly acquired disability was associated with 1.74 -fold (HR: $1.74,95 \%$ CI 1.31-2.33; $P<0.001)$ higher 2-year all-cause mortality, but decreased income $(P=0.571)$ and unemployment $(P=0.952)$ were not.

In model 3, newly acquired respiratory disability was associated with a 3.15-fold higher 2-year all-cause mortality (HR: $3.15,95 \%$ CI $1.89-5.24 ; P<0.001$ ). Table S5 shows the results of multivariable Cox regression models for 2-year respiratory and other mortality rates after ARDS 
Table 3 Multivariable logistic regression model for QOL worsening among ARDS survivors

\begin{tabular}{|c|c|c|}
\hline Variable & OR $(95 \% \mathrm{CI})$ & $P$ value \\
\hline Age, year & $0.99(0.99,0.99)$ & $<0.001$ \\
\hline Sex, male & $1.17(1.03,1.32)$ & 0.018 \\
\hline Admitting department: IM (vs non-IM) & $0.91(0.78,1.07)$ & 0.261 \\
\hline Total cost for hospitalization, USD, per 1000 USD & $1.01(1.01,1.02)$ & 0.022 \\
\hline \multicolumn{3}{|l|}{ Hospital admission } \\
\hline Transfer from another hospital & 1 & \\
\hline Admission through Emergency Room & $1.00(0.77,1.31)$ & 0.984 \\
\hline Admission through outpatient clinic & $0.90(0.68,1.18)$ & 0.434 \\
\hline \multicolumn{3}{|l|}{ Annual case volume of ARDS admission } \\
\hline Q2: 5-14 (vs Q1 $\leq 4)$ & $1.04(0.85,1.26)$ & 0.728 \\
\hline Q3: 15-28 (vs Q1 $\leq 4)$ & $0.95(0.79,1.14)$ & 0.579 \\
\hline $\mathrm{Q} 4 \geq 28(\mathrm{vs} \mathrm{Q} 1 \leq 4)$ & $1.11(0.91,1.34)$ & 0.296 \\
\hline \multicolumn{3}{|l|}{ Treatment result } \\
\hline Discharge, and follow-up in same hospital & 1 & \\
\hline Transfer to other long-term facility center & $0.74(0.58,0.95)$ & 0.017 \\
\hline Discharge, and outpatient clinic follow-up & $0.80(0.70,0.91)$ & 0.001 \\
\hline Main diagnosis of ARDS (vs secondary diagnosis of ARDS) & $0.92(0.81,1.04)$ & 0.184 \\
\hline Sepsis-associated ARDS & $1.03(0.85,1.26)$ & 0.742 \\
\hline Diagnosis of shock during hospitalization & $0.79(0.60,1.05)$ & 0.110 \\
\hline \multicolumn{3}{|l|}{$\mathrm{CCI}$ at hospital admission for ARDS treatment } \\
\hline $2-3($ vs $0-1)$ & $0.94(0.80,1.11)$ & 0.466 \\
\hline $4-5$ (vs $0-1)$ & $0.92(0.76,1.10)$ & 0.355 \\
\hline$\geq 6($ vs $0-1)$ & $1.12(0.92,1.36)$ & 0.249 \\
\hline ECMO support & $0.89(0.63,1.24)$ & 0.473 \\
\hline NMBA use & $0.98(0.84,1.15)$ & 0.815 \\
\hline CRRT use & $1.13(0.81,1.57)$ & 0.469 \\
\hline Duration of mechanical ventilator use, day & $1.00(0.99,1.01)$ & 0.428 \\
\hline Experience of CPR during hospitalization & $1.05(0.71,1.54)$ & 0.817 \\
\hline \multicolumn{3}{|l|}{ Year of admission for ARDS } \\
\hline 2011 (vs 2010) & $1.00(0.77,1.30)$ & 0.998 \\
\hline 2012 (vs 2010) & $1.34(1.03,1.74)$ & 0.027 \\
\hline 2013 (vs 2010) & $1.06(0.80,1.40)$ & 0.706 \\
\hline 2014 (vs 2010) & $1.04(0.81,1.36)$ & 0.744 \\
\hline 2015 (vs 2010) & $1.20(0.93,1.56)$ & 0.165 \\
\hline 2016 (vs 2010) & $1.20(0.94,1.53)$ & 0.139 \\
\hline 2017 (vs 2010) & $1.08(0.84,1.39)$ & 0.546 \\
\hline 2018 (vs 2010) & $1.46(1.13,1.87)$ & 0.003 \\
\hline
\end{tabular}

Hosmer Lemeshow test, Chi-square: $4.54, \mathrm{df}=8, P=0.806$

QOL, quality of life; OR, Odds ratio; CI, confidence interval; ARDS, acute respiratory distress syndrome; IM, internal medicine; CCI, Charlson comorbidity index; ECMO, extracorporeal membrane oxygenation; $\mathrm{NMB}$, neuromuscular blockade; CPR, cardiopulmonary resuscitation diagnosis among survivors. The newly acquired disability was not associated with 2-year respiratory mortality $(P=0.163)$, but newly acquired respiratory disability was associated with a 6.61-fold higher risk of 2-year respiratory mortality (HR: 6.61, 95\% CI 3.14-13.90; $P<0.001$ ). In addition, newly acquired disability and respiratory disability were associated with 1.80-fold (HR: 1.80, 95\% CI 1.30-2.49; $P<0.001$ ) and 2.09-fold (HR: $2.09,95 \%$
CI 1.03-4.26; $P=0.042$ ) higher risk of 2-year mortality, respectively. Figures $\mathrm{S} 1$ and $\mathrm{S} 2$ show the survival plots that were derived from the multivariable Cox regression model for 2-year all-cause mortality among ARDS survivors (Fig. S1: newly acquired disability group vs. others; Fig. S2: newly acquired respiratory disability group vs. others). 
Table 4 QOL worsening according to age, CCI, day of total mechanical ventilatory support, duration of hospitalization, use of ECMO support, sepsis-associated ARDS, and experience of CPR among ARDS survivors

\begin{tabular}{lr}
\hline Subgroups & $\begin{array}{r}\text { QOL worsening after } \\
\text { ARDS treatment } n(\%)\end{array}$ \\
\hline Age & \\
Age $\geq 66$-year-old & 722 of $2252(32.1)$ \\
Age $<66$-year-old & 945 of $2200(43.0)$ \\
CCI at ARDS & \\
$>3$ points & 652 of $1736(37.6)$ \\
$\leq 3$ points & 1015 of $2716(37.4)$ \\
Day of total mechanical ventilator support & \\
$\geq 2$ days & 662 of $1682(39.4)$ \\
$<2$ days & 1005 of $2770(36.3)$ \\
Duration of hospitalization at ARDS treatment & \\
$\geq 15$ days & 908 of $2367(38.4)$ \\
$<15$ days & 759 of $2085(36.4)$ \\
Use of ECMO support & \\
ECMO support & 101 of $215(47.0)$ \\
No ECMO support & 1566 of $4237(37.0)$ \\
Sepsis & \\
Sepsis-associated ARDS & \\
Non-sepsis-associated ARDS & 1566 of $4237(37.0)$ \\
CPR & 1456 of $3926(37.1)$ \\
Experience of CPR & \\
No experience of CPR & 52 of $116(44.8)$ \\
\hline
\end{tabular}

QOL, quality of life; CCI, Charlson comorbidity index; ARDS, acute respiratory distress syndrome; ECMO, extracorporeal membrane oxygenation; CPR, cardiopulmonary resuscitation

Table 5 Multivariable Cox regression model for 2-year mortality after diagnosis of ARDS

\begin{tabular}{lll}
\hline Variable & HR $(95 \%$ CI $)$ & $P$ value \\
\hline QOL worsening (model 1) & $1.10(0.92,1.78)$ & 0.140 \\
QOL worsening in detail (model 2) & & \\
Decreased income & $0.95(0.78,1.15)$ & 0.571 \\
Loss of job & $1.01(0.71,1.43)$ & 0.952 \\
Newly acquired disability & $1.74(1.31,2.33)$ & $<0.001$ \\
QOL worsening in detail (model 3) & & \\
Decreased income & $0.94(0.75,1.10)$ & 0.482 \\
Loss of job & $1.00(0.70,1.43)$ & 0.948 \\
Newly acquired respiratory disability & $3.15(1.89,5.24)$ & $<0.001$ \\
\hline
\end{tabular}

ARDS, acute respiratory distress syndrome; HR, hazard ratio; CI, confidence interval; QOL, quality of life

\section{Discussion}

In this population-based cohort study, we showed that almost one-third (37.4\%) of ARDS survivors experienced worsening QOL at the 1-year follow-up period after diagnosis of ARDS. Among the QOL factors, acquired disability was an independent risk factor for poorer 2-year survival, while unemployment and decreased household income level were not significantly associated poorer 2 -year survival. Interestingly, newly acquired respiratory disability was a more significant risk factor for poorer 2-year all-cause and respiratory mortality.

The newly acquired disability means that ARDS survivors had a persistent and functional disability in daily life due to ARDS after hospital discharge, as reported in a previous study [11]. We recently reported that newly acquired disability was a significant risk factor for poorer survival among survivors of sepsis [19] or ECMO therapy [20]. In addition to the previous reports [19, 20], our current results suggest that the newly acquired disability showed severe comorbid status and poor recovery from ARDS after hospital discharge. According to a study by Herridge et al., decreased QOL due to physical sequelae is a hallmark legacy in ARDS survivors [11]. We also showed that the decreased QOL due to newly acquired disability might worsen the long-term survival outcomes among ARDS survivors.

Interestingly, newly acquired respiratory disability was a significant risk factor for poorer 2-year all-cause and respiratory mortality. A pulmonologist in South Korea determined most respiratory disabilities, and we examined the criteria for determining respiratory disability (Table S1). According to the criteria, we can identify that the survivors with acquired disability suffered from severe restrictive patterns of respiratory disease with hypoxemia. Considering that chronic respiratory disease-related death is a substantial issue in Asian countries [21], the severe pattern of chronic respiratory diseases such as respiratory disability might affect poorer survival outcomes among South Korean ARDS survivors. In addition, we recently reported that newly developed chronic respiratory diseases were associated with higher 5-year mortality among survivors of ECMO therapy due to respiratory failure or ARDS [22]; thus, ARDS survivors who have a new respiratory disability might also be a high-risk group that needs special attention during healthcare utilization.

Regarding income level and unemployment, we found no significant association between these factors and mortality in this study. These results echo the associations between household income level and unemployment previously observed in survivors of sepsis and ECMO in South Korea [19, 20]. In South Korea, all individuals pay a fixed 
rate for health insurance premiums based on their income, with approximately $67 \%$ of their medical expenses being subsidized by the government [23]. However, those who cannot afford insurance premiums or have difficulty financially supporting themselves are included in the Medical Aid Program. In this program, the government covers almost all medical expenses to reduce the financial burden of medical costs [24]. Therefore, mortality was not influenced by unemployment or decreased household income levels among ARDS survivors in South Korea.

In the subgroup analysis, this study revealed that worsening QOL was more evident among ARDS survivors in the $<66$-year-old age group, $\geq 2$ days of total mechanical ventilator support group, and $\geq 15$ days of hospitalization of the ARDS treatment group, ECMO support group, and survivors who experienced CPR during hospitalization. Joblessness and lost earnings were significantly reported among ARDS survivors in the United States [12]. As people younger than 66 years might enjoy higher levels of employment and household income than those older than 66 years, the deterioration of QOL might occur more frequently in relatively younger ARDS survivors. Recently, prolonged duration of invasive mechanical ventilation was associated with worsening QOL among Coronavirus disease-related ARDS survivors in Italy [25]. Although the prolonged duration of mechanical ventilatory support was not associated with the prevalence of worsening QOL in multivariable model (Table 3), it was observed that QOL worsened in the $\geq 2$ days of total mechanical ventilator support group and $\geq 15$ days of hospitalization in the ARDS treatment group of this study. Therefore, the impact of these factors on worsening QOL among ARDS survivors should be confirmed in future study.

This study has several limitations. First, there were some variables we could not include because the NHIS database does not record $\mathrm{d}$ them, such as body mass index, $\mathrm{PaO}_{2} /$ $\mathrm{FiO}_{2}$ ratio, and Acute Physiologic Assessment and Chronic Health Evaluation II scores at admission for ARDS treatment. Second, we used registered ICD-10 codes for calculating CCI in this study, but the codes might differ from the actual underlying diseases. For example, some patients with mild liver disease could not be diagnosed using ICD10 codes due to their lack of access to healthcare resources. Third, some treatment options, such as prone positioning, were not included in this study because they do not have prescription codes in South Korea [26]. Fourth, we did not assess the severity of ARDS; thus, the survival analysis in this study should be carefully interpreted. Lastly, the generalizability of our findings might be limited because the public health insurance system in South Korea cannot be applied to other countries.

In conclusion, at the 1-year follow-up, one-third of ARDS survivors in South Korea experienced worsening
QOL. Specifically, newly acquired disability and respiratory disability were associated with a higher risk of 2-year allcause and respiratory mortality among ARDS survivors. Our results suggest that worsening of QOL is a serious concern and public health issue among ARDS survivors, and QOL might negatively affect long-term survival.

Supplementary Information The online version contains supplementary material available at https://doi.org/10.1007/s00540-022-03036-9.

Acknowledgements None.

Author contributions TKO designed the study, analyzed, and interpreted the data, and drafted the manuscript; IAS and HRC contributed to the study conceptualization, acquisition of data, and review of the manuscript. All the authors have provided approval for the final version of the manuscript.

Funding None.

\section{Declarations}

Conflict of interests The authors have no conflicts of interest.

\section{References}

1. Umbrello M, Formenti P, Bolgiaghi L, Chiumello D. Current concepts of ARDS: a narrative review. Int J Mol Sci. 2016. https:// doi.org/10.3390/ijms18010064.

2. Bellani G, Laffey JG, Pham T, Fan E, Brochard L, Esteban A, Gattinoni L, van Haren F, Larsson A, McAuley DF, Ranieri M, Rubenfeld G, Thompson BT, Wrigge H, Slutsky AS, Pesenti A, Investigators LS, Group ET. Epidemiology, patterns of care, and mortality for patients with acute respiratory distress syndrome in intensive care units in 50 countries. JAMA. 2016;315(8):788-800. https://doi.org/10.1001/jama.2016.0291.

3. Maca J, Jor O, Holub M, Sklienka P, Bursa F, Burda M, Janout V, Sevcik P. Past and present ARDS mortality rates: a systematic review. Respir Care. 2017;62(1):113-22. https://doi.org/10.4187/ respcare.04716.

4. Dowdy DW, Eid MP, Dennison CR, Mendez-Tellez PA, Herridge MS, Guallar E, Pronovost PJ, Needham DM. Quality of life after acute respiratory distress syndrome: a meta-analysis. Intensive Care Med. 2006;32(8):1115-24. https://doi.org/10.1007/ s00134-006-0217-3.

5. Davidson TA, Caldwell ES, Curtis JR, Hudson LD, Steinberg KP. Reduced quality of life in survivors of acute respiratory distress syndrome compared with critically ill control patients. JAMA. 1999;281(4):354-60. https://doi.org/10.1001/jama.281.4.354.

6. Granja C, Morujao E, Costa-Pereira A. Quality of life in acute respiratory distress syndrome survivors may be no worst than in other ICU survivors. Intensive Care Med. 2003;29(10):1744-50. https://doi.org/10.1007/s00134-003-1808-X.

7. Hopkins RO, Weaver LK, Collingridge D, Parkinson RB, Chan KJ, Orme JF Jr. Two-year cognitive, emotional, and quality-oflife outcomes in acute respiratory distress syndrome. Am J Respir Crit Care Med. 2005;171(4):340-7. https://doi.org/10.1164/rccm. 200406-763OC.

8. Masclans JR, Roca O, Munoz X, Pallisa E, Torres F, Rello J, Morell F. Quality of life, pulmonary function, and tomographic 
scan abnormalities after ARDS. Chest. 2011;139(6):1340-6. https://doi.org/10.1378/chest.10-2438.

9. Apfelbacher C, Brandstetter S, Blecha S, Dodoo-Schittko F, Brandl M, Karagiannidis C, Quintel M, Kluge S, Putensen C, Bercker S, Ellger B, Kirschning T, Arndt C, Meybohm P, WeberCarstens S, Group DS, Bein T. Influence of quality of intensive care on quality of life/return to work in survivors of the acute respiratory distress syndrome: prospective observational patient cohort study (DACAPO). BMC Public Health. 2020;20(1):861. https://doi.org/10.1186/s12889-020-08943-8.

10. Herridge MS, Moss M, Hough CL, Hopkins RO, Rice TW, Bienvenu OJ, Azoulay E. Recovery and outcomes after the acute respiratory distress syndrome (ARDS) in patients and their family caregivers. Intensive Care Med. 2016;42(5):725-38. https://doi. org/10.1007/s00134-016-4321-8.

11. Herridge MS, Tansey CM, Matte A, Tomlinson G, Diaz-Granados N, Cooper A, Guest CB, Mazer CD, Mehta S, Stewart TE, Kudlow P, Cook D, Slutsky AS, Cheung AM, Canadian Critical Care Trials G. Functional disability 5 years after acute respiratory distress syndrome. N Engl J Med. 2011;364(14):1293-304. https://doi.org/ 10.1056/NEJMoa1011802.

12. Kamdar BB, Huang M, Dinglas VD, Colantuoni E, von Wachter TM, Hopkins RO, Needham DM, National Heart L, Blood Institute Acute Respiratory Distress Syndrome N. Joblessness and lost earnings after acute respiratory distress syndrome in a 1-year National Multicenter Study. Am J Respir Crit Care Med. 2017;196(8):1012-20. https://doi.org/10.1164/rccm. 201611-2327OC.

13. Kamdar BB, Sepulveda KA, Chong A, Lord RK, Dinglas VD, Mendez-Tellez PA, Shanholtz C, Colantuoni E, von Wachter TM, Pronovost PJ, Needham DM. Return to work and lost earnings after acute respiratory distress syndrome: a 5-year prospective, longitudinal study of long-term survivors. Thorax. 2018;73(2):125-33. https://doi.org/10.1136/thoraxjnl-2017-210217.

14. Brown SM, Wilson E, Presson AP, Zhang C, Dinglas VD, Greene T, Hopkins RO, Needham DM, with the National Institutes of Health NAN. Predictors of 6-month health utility outcomes in survivors of acute respiratory distress syndrome. Thorax. 2017;72(4):311-7. https://doi.org/10.1136/thora xjnl-2016-208560.

15. von Elm E, Altman DG, Egger M, Pocock SJ, Gotzsche PC, Vandenbroucke JP, Initiative S. The Strengthening the Reporting of Observational Studies in Epidemiology (STROBE) Statement: guidelines for reporting observational studies. Int J Surg. 2014;12(12):1495-9. https://doi.org/10.1016/j.ijsu.2014.07.013.

16. Force ADT, Ranieri VM, Rubenfeld GD, Thompson BT, Ferguson ND, Caldwell E, Fan E, Camporota L, Slutsky AS. Acute respiratory distress syndrome: the Berlin definition. JAMA. 2012;307(23):2526-33. https://doi.org/10.1001/jama.2012.5669.

17. Sim YS, Lee JH, Lee WY, Suh DI, Oh YM, Yoon JS, Lee JH, Cho JH, Kwon CS, Chang JH. Spirometry and bronchodilator test.
Tuberc Respir Dis (Seoul). 2017;80(2):105-12. https://doi.org/ 10.4046/trd.2017.80.2.105.

18. Ike JD, Kempker JA, Kramer MR, Martin GS. The association between acute respiratory distress syndrome hospital case volume and mortality in a U.S. Cohort, 2002-2011. Crit Care Med. 2018;46(5):764-73. https://doi.org/10.1097/CCM.0000000000 003015 .

19. Oh TK, Song IA. Quality of life after sepsis and its association with mortality among sepsis survivors in South Korea: a population level cohort study. J Crit Care. 2021;64:193-8. https://doi. org/10.1016/j.jcrc.2021.04.018.

20. Cho H-W, Song I-A, Oh TK. Quality of life and long-term mortality among survivors of extracorporeal membrane oxygenation: a nationwide cohort study in South Korea. Crit Care Med. 2021.

21. Baptista EA, Dey S, Pal S. Chronic respiratory disease mortality and its associated factors in selected Asian countries: evidence from panel error correction model. BMC Public Health. 2021;21(1):53. https://doi.org/10.1186/s12889-020-10042-7.

22. Oh TK, Cho HW, Lee HT, Song IA. Chronic respiratory disease and survival outcomes after extracorporeal membrane oxygenation. Respir Res. 2021;22(1):195. https://doi.org/10.1186/ s12931-021-01796-8.

23. Song YJ. The South Korean health care system. JMAJ. 2009;52(3):206-9.

24. Lee DW, Jang J, Choi DW, Jang SI, Park EC. The effect of shifting medical coverage from National Health Insurance to Medical Aid type I and type II on health care utilization and out-of-pocket spending in South Korea. BMC Health Serv Res. 2020;20(1):979. https://doi.org/10.1186/s12913-020-05778-2.

25. Gamberini L, Mazzoli CA, Sintonen H, Colombo D, Scaramuzzo G, Allegri D, Tonetti T, Zani G, Capozzi C, Giampalma E, Agnoletti V, Becherucci F, Bertellini E, Castelli A, Cappellini I, Cavalli I, Crimaldi F, Damiani F, Fusari M, Gordini G, Laici C, Lanza MC, Leo M, Marudi A, Nardi G, Ottaviani I, Papa R, Potalivo A, Ranieri VM, Russo E, Taddei S, Volta CA, Spadaro S, Collaboration I-RC. Quality of life of COVID-19 critically ill survivors after ICU discharge: 90 days follow-up. Qual Life Res. 2021;30(10):2805-17. https://doi.org/10.1007/ s11136-021-02865-7.

26. Scholten EL, Beitler JR, Prisk GK, Malhotra A. Treatment of ARDS with prone positioning. Chest. 2017;151(1):215-24. https://doi.org/10.1016/j.chest.2016.06.032.

Publisher's Note Springer Nature remains neutral with regard to jurisdictional claims in published maps and institutional affiliations. 